\title{
Status of the Unitarity Triangle in the Standard Model
}

\author{
Marcella Bona* \\ INFN and Università di Torino, Italy \\ E-mail: bona@to.infn.it
}

on behalf of $\mathbf{U T}$ fit Collaboration ${ }^{\dagger}$

\begin{abstract}
We review here the status of the Unitarity Triangle in the Standard Model. Within the Standard Model (SM), all flavour and CP violating quark weak interactions are governed by the CKM matrix, which can be parameterized in terms of three angles and one phase, or, more conveniently, in terms of the parameters $\lambda, A, \bar{\rho}$ and $\bar{\eta}$. This implies very strong correlations among flavour and CP violating observables within the SM. The Unitarity Triangle (UT) is a very useful tool to analyze these correlations. With the recent data on $B$ decays, the UT fit has become strongly overconstrained, thus it is now possible to test the CKM mechanism within the SM and to derive constraints on New Physics (NP). In this talk, we will review the first aspect, while the second is contained in ref. [1]. More details on the procedure and on the results can be found in ref. [2].
\end{abstract}

International Europhysics Conference on High Energy Physics

July 21 st - 27th 2005

Lisboa, Portugal

* Speaker.

${ }^{\dagger}$ UTfit Collaboration: M. Bona, M. Ciuchini, E. Franco, V. Lubicz, G. Martinelli, F. Parodi, M. Pierini, P. Roudeau, C. Schiavi, L. Silvestrini, A. Stocchi and V. Vagnoni 


\section{The Standard Model Unitarity Triangle analysis}

Using the latest determinations of several theoretical and experimental parameters, we update the Unitarity Triangle analysis in the Standard Model. The basic experimental constraints come from the measurements of $\left|V_{u b} / V_{c b}\right|, \Delta m_{d}$, the lower limit on $\Delta m_{s}, \varepsilon_{K}$, and the measurement of the phase of the $B_{d}-\bar{B}_{d}$ mixing amplitude through the time-dependent $\mathrm{CP}$ asymmetry in $B^{0} \rightarrow J / \psi K^{0}$ decays. In addition, we consider the direct determination of $\alpha, \gamma, 2 \beta+\gamma$ and $\cos 2 \beta$ from the measurements of new CP-violating quantities, recently performed at the $B$ factories. The results and the plots presented in this paper can also be found at the URL http://www . utfit.org, where they are continuously updated with the newest experimental and theoretical results.

The values and errors of the relevant quantities used as inputs in the standard analysis of the CKM parameters are summarized in Table 1. Additional inputs corresponding to the measurements of the angles $\gamma$ and $\alpha$ can be found in ref. [4], while ref. [2] describes the procedure followed to extract these constraints from experimental data.

Table 1: Values of the relevant quantities used in the UT fit.

\begin{tabular}{|c|c|c|c|}
\hline Parameter & Value & Gaussian & Uniform \\
\hline$\lambda$ & 0.2258 & 0.0014 & - \\
\hline$\left|V_{c b}\right|$ (excl.) & $41.4 \cdot 10^{-3}$ & $2.1 \cdot 10^{-3}$ & - \\
\hline$\left|V_{c b}\right|$ (incl.) & $41.6 \cdot 10^{-3}$ & $0.7 \cdot 10^{-3}$ & $0.6 \cdot 10^{-3}$ \\
\hline$\left|V_{u b}\right|$ (excl.) & $38.0 \cdot 10^{-4}$ & $2.7 \cdot 10^{-4}$ & $4.7 \cdot 10^{-4}$ \\
\hline$\left|V_{u b}\right|$ (incl.) & $43.9 \cdot 10^{-4}$ & $2.0 \cdot 10^{-4}$ & $2.7 \cdot 10^{-4}$ \\
\hline$\Delta m_{d}$ & $0.502 \mathrm{ps}^{-1}$ & $0.006 \mathrm{ps}^{-1}$ & - \\
\hline$\Delta m_{s}$ & $>14.5 \mathrm{ps}^{-1}$ & @ 95\% C.L. & \\
\hline$f_{B_{s}} \sqrt{\hat{B}_{B_{s}}}$ & $276 \mathrm{MeV}$ & $38 \mathrm{MeV}$ & - \\
\hline$\xi$ & 1.24 & 0.04 & 0.06 \\
\hline$\hat{B}_{K}$ & 0.79 & 0.04 & 0.09 \\
\hline$\varepsilon_{K}$ & $2.28 \cdot 10^{-3}$ & $1.3 \cdot 10^{-5}$ & - \\
\hline$f_{K}$ & $159 \mathrm{MeV}$ & \multicolumn{2}{|c|}{ fi xed } \\
\hline $\sin 2 \beta$ & 0.687 & 0.032 & - \\
\hline $\bar{m}_{t}$ & $165.0 \mathrm{GeV}$ & $3.9 \mathrm{GeV}$ & - \\
\hline $\bar{m}_{b}$ & $4.21 \mathrm{GeV}$ & $0.08 \mathrm{GeV}$ & - \\
\hline $\bar{m}_{c}$ & $1.3 \mathrm{GeV}$ & $0.1 \mathrm{GeV}$ & - \\
\hline$\alpha_{s}\left(M_{Z}\right)$ & 0.119 & 0.003 & - \\
\hline
\end{tabular}

The main novelty in the last two years in the UT analysis is the measurement of the angles of the UT at the $B$ factories. While $\sin 2 \beta$ is by now part of the "classic" fit, it is only recently that the measurements of the CP asymmetry in $B \rightarrow J / \psi K^{*}$ (of $B \rightarrow D^{0} h^{0}$ decays) have provided a determination of $\cos 2 \beta(\beta)$. These determinations and the corresponding constraints on the $\bar{\rho}-\bar{\eta}$ plane are shown in Fig. 1. It is evident how these additional measurements can suppress one of the two bands determined by $\sin 2 \beta$.

The angle $\gamma$ can be determined studying the interference of $b \rightarrow u$ and $b \rightarrow c$ transitions in $B \rightarrow D^{(*)} K^{(*)}$ decays, using the GLW, ADS or Dalitz methods. The resulting p.d.f. for $\gamma$ is reported 

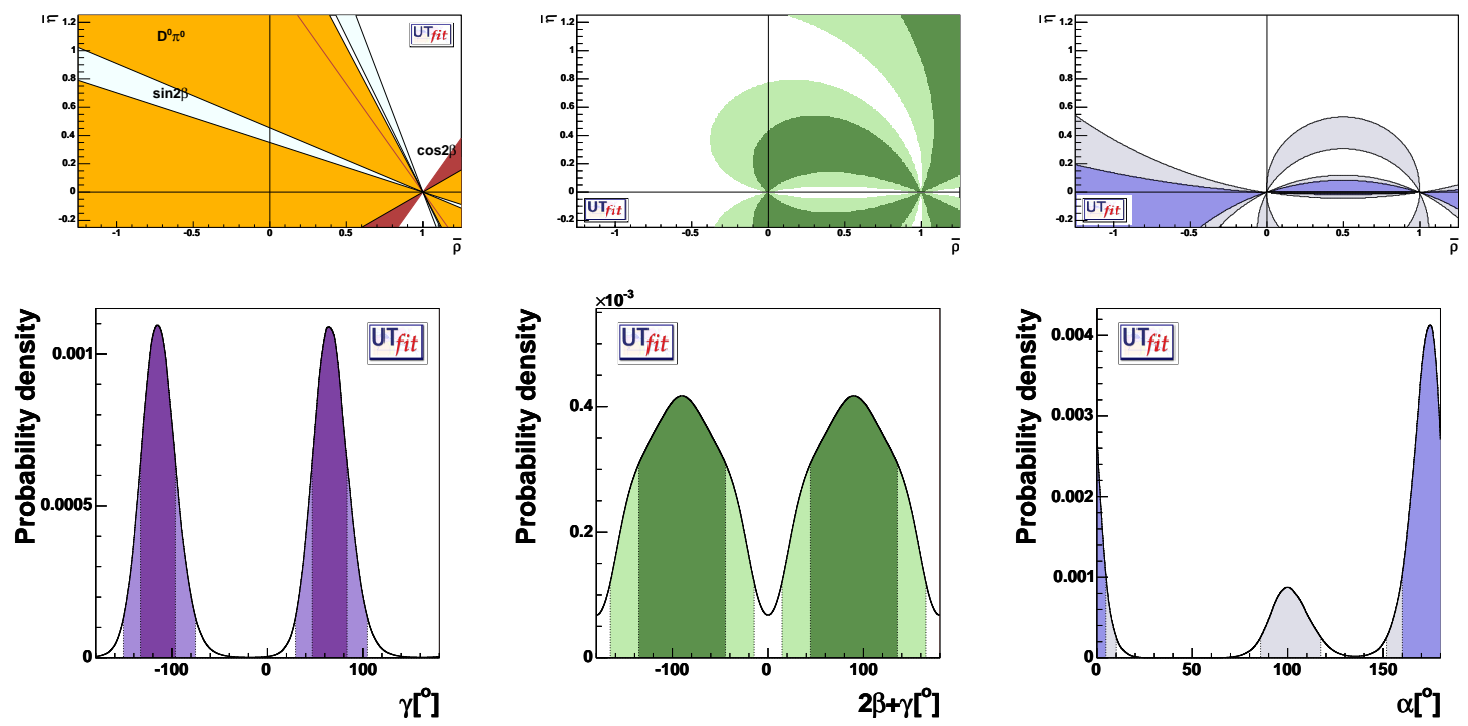

Figure 1: Top-left plot: The regions in the $\bar{\rho}-\bar{\eta}$ plane determined at $2 \sigma$ probability by the measurements of $\cos 2 \beta$ and $\beta$. For reference, the region selected by $\sin 2 \beta$ is also reported. Bottom-left plot: P.d.f. for $\gamma$ using all available information. Middle plots: P.d.f. for $2 \beta+\gamma$ and its impact on the $\bar{\rho}-\bar{\eta}$ plane. Right plots: P.d.f. for $\alpha$ and its impact on the $\bar{\rho}-\bar{\eta}$ plane.

in Fig. 1.

Studying $B^{0} \rightarrow D^{(*)} \pi(\rho)$ decays, it is possible to extract $\sin (2 \beta+\gamma)$ from the time-dependent $\mathrm{CP}$ asymmetries. However, present data are insuffi cient to allow this determination, so that additional input is needed. This can come from SU(3)-related $B \rightarrow D_{s}$ channels, if one neglects annihilation contributions. The total theoretical error in this procedure can be conservatively estimated around $100 \%$ [5]. Including this error, we obtain the constraint on $\sin (2 \beta+\gamma)$ shown in Fig. 1, together with the impact on the $\bar{\rho}-\bar{\eta}$ plane.

The angle $\alpha$ can be extracted from the time-dependent $\mathrm{CP}$ asymmetry in $B \rightarrow \pi \pi, \rho \pi, \rho \rho$ decays, with the uncertainty related to penguin pollution. Given the presently unclear experimental situation and the large penguin pollution, we do not consider here $B \rightarrow \pi \pi$ decays. The determination of $\alpha$ from the other modes using the $B$-factory data and the corresponding constraint on the $\bar{\rho}$ $-\bar{\eta}$ plane are reported in Fig. 1.

Using the angle measurements described above, it is possible to obtain a determination of the UT with an accuracy comparable to the determination obtained using all the other measurements (see Fig. 2). The UT fi $t$ is therefore now strongly overconstrained, and it tests in a highly nontrivial way the CKM picture of fhvour and CP violation.

Combining all available information, we obtain the "state of the art" determination in Fig. 2, and the results for UT parameters reported in Table 2. Comparing the results of the fit with the input values, there is a small $(<2 \sigma)$ discrepancy in the values of $\sin 2 \beta$ and $\left|V_{u b}\right|$ from inclusive decays. 
Table 2: Values and probability ranges for the UT parameters obtained from the UT fit using all constraints.

\begin{tabular}{ccc}
\hline & $68 \%$ & $95 \%$ \\
$\bar{\rho}$ & $0.208 \pm 0.036$ & {$[0.135,0.277]$} \\
$\bar{\eta}$ & $0.347 \pm 0.021$ & {$[0.306,0.388]$} \\
$\alpha\left[^{\circ}\right]$ & $97.1 \pm 5.6$ & {$[86.0,107.7]$} \\
$\beta\left[^{\circ}\right]$ & $23.8 \pm 1.4$ & {$[21.3,26.2]$} \\
$\left.\gamma^{\circ}\right]$ & $58.9 \pm 5.4$ & {$[48.7,69.9]$} \\
$\sin 2 \beta$ & $0.736 \pm 0.023$ & {$[0.690,0.781]$} \\
$\left|V_{u b}\right|\left[10^{-4}\right]$ & $38.5 \pm 1.4$ & {$[35.7,41.4]$} \\
\hline
\end{tabular}
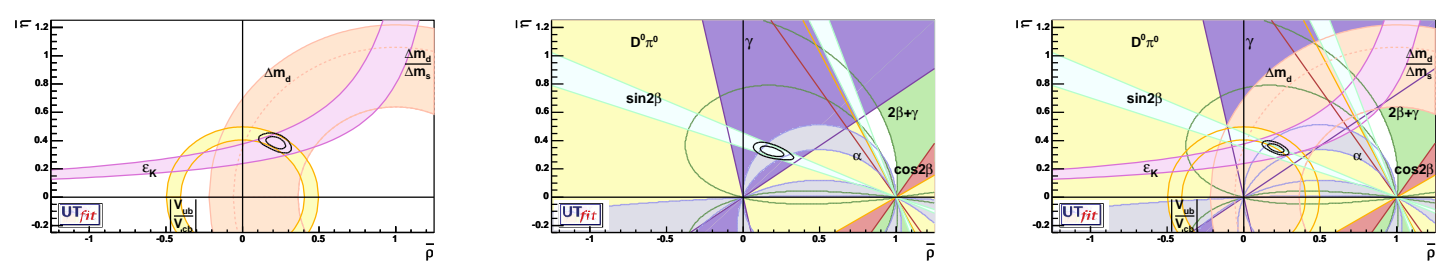

Figure 2: From left to right: Determinations of the UT without using angle measurements (left), using only angles (middle) and using all information (right).

\section{Conclusions}

Flavour physics in the quark sector has entered its mature age. Today the Unitarity Triangle parameters are known with good precision. This test of the SM has been allowed by the impressive improvements achieved on non-perturbative methods which have been used to extract the CKM parameters. Many $B$ decay branching ratios and $C P$ asymmetries have been measured at $B$ factories. The outstanding result is the determination of $\sin 2 \beta$ from $B$ hadronic decays into charmonium- $K^{0}$ fi nal states. Many other exclusive hadronic rare $B$ decays have been measured and constitute a gold mine for weak and hadronic physics, allowing in principle to extract different combinations of the Unitarity Triangle angles. Besides presenting an update of the standard UTfit analysis, we have shown here that new measurements at $B$ factories begin to have an impact on the overall picture of the Unitarity Triangle determination. In particular the angle $\gamma$ is today measured through charged $B$ decays into $D K$ fi nal states within $20 \%$ accuracy and only a twofold ambiguity. In the following years the precise measurements of the UT angles will provide further tests of the Standard Model in the flavour sector to an accuracy up to the percent level.

\section{References}

[1] M. Bona [on behalf of the $\mathbf{U} \mathbf{f} i t$ Collaboration], talk given at this conference with the title "Testing New Physics with Unitarity Triangle Fit”

[2] M. Bona et al. [UT $f t$ Collaboration], JHEP 0507 (2005) 028. and references therein

[3] M. Bona et al. [UI $f i t$ Collaboration], arXiv:hep-ph/0509219.

[4] The Heavy Flavour Averaging Group, summer 2005 averages.

[5] D. Pirjol, talk at the 10th International Conference on B-Physics at Hadron Machines (BEAUTY 2005), June 20th-24th 2005, Assisi, Italy. 